\title{
ON CERTAIN ENUMERATION PROBLEMS IN TWO-DIMENSIONAL TOPOLOGY
}

\author{
VLADimir TURAeV
}

\begin{abstract}
We announce a solution to several enumeration problems in topology of surfaces. This includes an enumeration of homotopy classes of sections of locally trivial fiber bundles over surfaces and a computation of non-abelian 1-cohomology of surfaces.
\end{abstract}

\section{Introduction}

We announce a solution to several enumeration problems in two-dimensional topology. One problem deals with an arbitrary locally trivial fiber bundle $p: E \rightarrow W$ over a closed connected oriented surface $W$. The bundle $p$ may have sections, i.e., continuous mappings $s: W \rightarrow E$ such that $p s=\mathrm{id}_{W}$. If the fiber $F$ of $p$ is path-connected and the group $\pi_{1}(F)$ is finite, then the number of sections of $p$, considered up to homotopy and a natural action of $\pi_{2}(F)$, is finite. We give a formula computing this number in terms of certain 2-dimensional cohomology classes associated with irreducible complex linear representations of $\pi_{1}(F)$. This yields, in particular, the following solution to the existence problem for sections: the bundle $p$ has a section if and only if the integer number produced by our formula is non-zero. Note that the integer in question is always non-negative and is computable provided one has an efficient description of both the set of equivalence classes of irreducible representations of $\pi_{1}(F)$ and the action of $\pi_{1}(W)$ on this set determined by $p$. As a specific application, note the following theorem: in the case where the group $\pi_{1}(F)$ is abelian (and finite), the bundle $p: E \rightarrow W$ has a section if and only if the induced homomorphism $p_{*}: H_{2}(E) \rightarrow H_{2}(W)$ is surjective. In particular, if $p: E \rightarrow W$ is a principal $H$-bundle, where $H$ is a connected topological group with finite fundamental group, then $p$ is trivial if and only if $p_{*}\left(H_{2}(E)\right)=H_{2}(W)$.

The enumeration problem for the sections of $E \rightarrow W$ can be reformulated in terms of sections of the induced homomorphism $\pi_{1}(E) \rightarrow \pi_{1}(W)$. This generalizes to the following question. Given a group epimorphism $G^{\prime} \rightarrow G$ with finite kernel $\Gamma$ and a homomorphism $g: \pi_{1}(W) \rightarrow G$, calculate the number of lifts of $g$ to $G^{\prime}$. The finiteness of $\Gamma$ ensures that this number is finite (possibly, zero). Our main result computes this number in terms of 2-dimensional cohomology classes associated with irreducible representations of $\Gamma$. This encompasses the problem of finding whether or not $g$ lifts to $G^{\prime}$.

Other enumeration problems considered here deal with counting principal fiber bundles over $W$ and with a computation of non-abelian 1-cohomology of $W$.

Throughout this paper, we fix two (discrete) groups $G, G^{\prime}$ and an epimorphism $q: G^{\prime} \rightarrow G$ with finite kernel $\Gamma=\operatorname{Ker} q$. The symbol $W$ denotes a closed connected

Received by the editors April 9, 2008. 
oriented surface of positive genus with fundamental group $\pi$. My work on this paper was partially supported by the NSF grant DMS-0707078.

\section{Enumeration of homomorphisms}

By a (linear) representation of $\Gamma$, we mean a homomorphism $\Gamma \rightarrow G L_{n}(\mathbb{C})$ with $n=1,2, \ldots$ Two representations $\rho: \Gamma \rightarrow G L_{n}(\mathbb{C})$ and $\rho^{\prime}: \Gamma \rightarrow G L_{n^{\prime}}(\mathbb{C})$ are equivalent if $n=n^{\prime}$ and there is a matrix $M \in G L_{n}(\mathbb{C})$ such that $\rho^{\prime}(h)=M^{-1} \rho(h) M$ for all $h \in \Gamma$. A representation $\Gamma \rightarrow G L_{n}(\mathbb{C})$ is irreducible if the only linear subspaces of $\mathbb{C}^{n}$ preserved under the induced action of $\Gamma$ are 0 and $\mathbb{C}^{n}$. The set of equivalence classes of irreducible representations of $\Gamma$ is denoted $\operatorname{Irr}(\Gamma)$.

Since $\Gamma=\operatorname{Ker} q$ is a normal subgroup of $G^{\prime}$, the group $G^{\prime}$ acts on $\Gamma$ by conjugations. The induced action of $G^{\prime}$ on $\operatorname{Irr}(\Gamma)$ is trivial on $\Gamma \subset G^{\prime}$ and therefore induces an action of $G=G^{\prime} / \Gamma$ on $\operatorname{Irr}(\Gamma)$. Given an irreducible representation $\rho: \Gamma \rightarrow G L_{n}(\mathbb{C})$, let $G_{\rho} \subset G$ be the stabilizer of the equivalence class of $\rho$ under this action of $G$ on $\operatorname{Irr}(\Gamma)$. Thus, $G_{\rho}$ consists of all $\alpha \in G$ such that for some $\widetilde{\alpha} \in q^{-1}(\alpha)$, the representation $\Gamma \rightarrow G L_{n}(\mathbb{C}), h \mapsto \rho\left(\widetilde{\alpha}^{-1} h \widetilde{\alpha}\right)$ is equivalent to $\rho$. Note that if the latter condition holds for some $\widetilde{\alpha} \in q^{-1}(\alpha)$, then it holds for all $\widetilde{\alpha} \in q^{-1}(\alpha)$. The subgroup $G_{\rho}$ of $G$ depends only on the equivalence class of $\rho$.

The representation $\rho$ determines a cohomology class $\zeta_{\rho} \in H^{2}\left(G_{\rho} ; \mathbb{C}^{*}\right)$ as follows. For each $\alpha \in G_{\rho}$, pick $\widetilde{\alpha} \in q^{-1}(\alpha) \subset G^{\prime}$. Then there is a matrix $M_{\alpha} \in G L_{n}(\mathbb{C})$ such that

$$
\rho\left(\widetilde{\alpha}^{-1} h \widetilde{\alpha}\right)=M_{\alpha}^{-1} \rho(h) M_{\alpha}
$$

for all $h \in \Gamma$. The irreducibility of $\rho$ implies that $M_{\alpha}$ is unique up to multiplication by an element of $\mathbb{C}^{*}$; we fix $M_{\alpha}$ for all $\alpha \in G_{\rho}$. For any $\alpha, \beta \in G_{\rho}$, we have $\widetilde{\alpha \beta}^{-1} \widetilde{\alpha} \widetilde{\beta} \in \Gamma$. It is easy to check that there is a unique $\zeta_{\alpha, \beta} \in \mathbb{C}^{*}$ such that

$$
\zeta_{\alpha, \beta} M_{\alpha} M_{\beta}=M_{\alpha \beta} \rho\left(\widetilde{\alpha \beta}^{-1} \widetilde{\alpha} \widetilde{\beta}\right) \text {. }
$$

The family $\left\{\zeta_{\alpha, \beta}\right\}_{\alpha, \beta}$ turns out to be a 2-cocycle on $G_{\rho}$. Its cohomology class $\zeta_{\rho} \in$ $H^{2}\left(G_{\rho} ; \mathbb{C}^{*}\right)$ depends only on the equivalence class of $\rho$ and not on the choice of $\left\{M_{\alpha}\right\}_{\alpha}$ or $\{\widetilde{\alpha}\}_{\alpha}$. (The definitions of $G_{\rho}$ and $\zeta_{\rho}$ do not use the finiteness of $\Gamma$.)

Given a homomorphism $g: \pi=\pi_{1}(W) \rightarrow G$, a lift of $g$ to $G^{\prime}$ is a homomorphism $g^{\prime}: \pi \rightarrow G^{\prime}$ such that $q g^{\prime}=g$. The set (possibly empty) of all such lifts is denoted by $\operatorname{Hom}_{g}\left(\pi, G^{\prime}\right)$. Since $\pi$ is finitely generated and $\Gamma$ is finite, the set $\operatorname{Hom}_{g}\left(\pi, G^{\prime}\right)$ is

finite. The number of its elements is bounded from above by $|\Gamma|^{b}$, where $b$ is the first Betti number of $W$ and the vertical bars stand for the cardinality of a set. We now compute $\left|\operatorname{Hom}_{g}\left(\pi, G^{\prime}\right)\right|$ in terms of representations of $\Gamma$. Note that the finiteness of $\Gamma$ guarantees that the set $\operatorname{Irr}(\Gamma)$ is finite.

Theorem 1.1. Let $g: \pi=\pi_{1}(W) \rightarrow G$ be a group homomorphism. Then

$$
\left|\operatorname{Hom}_{g}\left(\pi, G^{\prime}\right)\right|=|\Gamma| \sum_{\rho \in \operatorname{Irr}(\Gamma), G_{\rho} \supset g(\pi)}\left(\frac{|\Gamma|}{\operatorname{dim} \rho}\right)^{-\chi(W)} g^{*}\left(\zeta_{\rho}\right)([W]),
$$

where $\rho$ ranges over the equivalence classes of irreducible (complex) representations of $\Gamma$ such that $G_{\rho} \supset g(\pi)$, and $g^{*}\left(\zeta_{\rho}\right)([W]) \in \mathbb{C}^{*}$ is the evaluation of $g^{*}\left(\zeta_{\rho}\right) \in H^{2}\left(\pi ; \mathbb{C}^{*}\right)$ on the fundamental class $[W] \in H_{2}(W)=H_{2}(\pi)$ of $W$. 
Here and below the unspecified group of coefficients in homology is $\mathbb{Z}$. The cohomology class $g^{*}\left(\zeta_{\rho}\right)$ is well-defined because $g(\pi) \subset G_{\rho}$. The addition on the right-hand side of $(1.2)$ is the addition in $\mathbb{C}$. The sum on the right-hand side of (1.2) is always non-empty because the trivial one-dimensional representation $\rho_{0}: \Gamma \rightarrow\{1\} \in \mathbb{C}^{*}=$ $G L_{1}(\mathbb{C})$ satisfies $G_{\rho_{0}}=G \supset g(\pi)$ and $g^{*}\left(\zeta_{\rho_{0}}\right)([W])=1$.

Note a few cases where Formula (1.2) is known or may be directly deduced from the known results. If $g=1$, then $\operatorname{Hom}_{g}\left(\pi, G^{\prime}\right)=\operatorname{Hom}(\pi, \Gamma)$. In this case $G_{\rho} \supset g(\pi)$ and $g^{*}\left(\zeta_{\rho}\right)([W])=1$ for all irreducible representations $\rho$ of $\Gamma$. Formula (1.2) is then equivalent to the well-known Frobenius-Mednykh formula

$$
|\operatorname{Hom}(\pi, \Gamma)|=|\Gamma| \sum_{\rho \in \operatorname{Irr}(\Gamma)}\left(\frac{|\Gamma|}{\operatorname{dim} \rho}\right)^{-\chi(W)} .
$$

For $W=S^{2}$, this formula is equivalent to the classical equality $\sum_{\rho}(\operatorname{dim} \rho)^{2}=|\Gamma|$. For $W=S^{1} \times S^{1}$, Formula (1.3) was first established by Frobenius [Fr]. The general case of (1.3) is due to Mednykh [Me], see also [FQ] and [Jo].

If $G^{\prime}=\Gamma \times G$ and $q: G^{\prime} \rightarrow G$ is the projection, then (1.2) directly follows from (1.3) since in this case $\operatorname{Hom}_{g}\left(\pi, G^{\prime}\right)=\operatorname{Hom}(\pi, \Gamma)$ and $\zeta_{\rho}$ is trivial for all $\rho$.

If $\Gamma=\operatorname{Ker} q$ is central in $G^{\prime}$, then Formula (1.2) is essentially obvious. In this case $G_{\rho}=G$ and $\operatorname{dim} \rho=1$ for all $\rho$, while $\zeta_{\rho}$ is the image of the standard cohomology class $\zeta \in H^{2}(G ; \Gamma)$ determined by $q$ under the coefficient homomorphism $H^{2}(G ; \Gamma) \rightarrow$ $H^{2}\left(G ; \mathbb{C}^{*}\right)$ induced by $\rho: \Gamma \rightarrow \mathbb{C}^{*}$. Formula $(1.2)$ can be deduced then from the following easy assertions: $g$ has a lift to $G^{\prime}$ if and only if $g^{*}(\zeta)=1$; if there are such lifts, then their number is equal to $|\Gamma|^{2-\chi(W)}$.

A proof of Theorem 1.1 in the full generality uses the techniques of topological quantum field theory and will be given elsewhere. Note that Formula (1.3) extends to surfaces with boundary and to non-orientable surfaces, see [Jo], [Sn]. Theorem 1.1 admits similar extensions, but we shall not discuss them here.

We keep the assumptions of Theorem 1.1 and establish several corollaries. Observe that

$$
g^{*}\left(\zeta_{\rho}\right)([W])=\left(\left.\zeta_{\rho}\right|_{g(\pi)}\right)\left(g_{*}([W])\right),
$$

where $\left.\zeta_{\rho}\right|_{g(\pi)} \in H^{2}\left(g(\pi) ; \mathbb{C}^{*}\right)$ is the restriction of $\zeta_{\rho} \in H^{2}\left(G_{\rho} ; \mathbb{C}^{*}\right)$ to $g(\pi)$ and

$$
g_{*}: H_{2}(W)=H_{2}(\pi) \rightarrow H_{2}(g(\pi))
$$

is the homomorphism induced by $g$. Formula (1.2) can be rewritten as

$$
\left|\operatorname{Hom}_{g}\left(\pi, G^{\prime}\right)\right|=|\Gamma| \sum_{\rho \in \operatorname{Irr}(\Gamma), G_{\rho} \supset g(\pi)}(|\Gamma| / \operatorname{dim} \rho)^{-\chi(W)}\left(\left.\zeta_{\rho}\right|_{g(\pi)}\right)\left(g_{*}([W])\right) .
$$

This implies the following claim.

Corollary 1.2. The number $\left|\operatorname{Hom}_{g}\left(\pi, G^{\prime}\right)\right|$ is determined by the homomorphism $q$ : $G^{\prime} \rightarrow G$, the genus of $W$, the group $g(\pi) \subset G$, and the homology class $g_{*}([W]) \in$ $H_{2}(g(\pi))$.

Note the following special case of (1.4).

Corollary 1.3. If $g: \pi \rightarrow G$ is an epimorphism, then

$$
\left|\operatorname{Hom}_{g}\left(\pi, G^{\prime}\right)\right|=|\Gamma| \sum_{\rho \in \operatorname{Irr}(\Gamma), G_{\rho}=G}(|\Gamma| / \operatorname{dim} \rho)^{-\chi(W)} \zeta_{\rho}\left(g_{*}([W])\right) .
$$


To proceed, we need the following property of $\zeta_{\rho}$. Consider the group $G_{\rho}^{\prime}=$ $q^{-1}\left(G_{\rho}\right) \subset G^{\prime}$. Taking determinant on both sides of $(1.1)$, one easily obtains that

$$
q^{*}\left((\operatorname{dim} \rho) \zeta_{\rho}\right)=0,
$$

where $q^{*}: H^{2}\left(G_{\rho} ; \mathbb{C}^{*}\right) \rightarrow H^{2}\left(G_{\rho}^{\prime} ; \mathbb{C}^{*}\right)$ is the homomorphism induced by $q$. This implies that the values of $\zeta_{\rho}$ on the image of the homomorphism $q_{*}: H_{2}\left(G_{\rho}^{\prime}\right) \rightarrow$ $H_{2}\left(G_{\rho}\right)$ are roots of unity of $\operatorname{order} \operatorname{dim} \rho$. The finiteness of $\Gamma$ implies that the image of $q_{*}$ is a subgroup of finite order in $H_{2}\left(G_{\rho}\right)$. Hence the values of $\zeta_{\rho}$ on all elements of $H_{2}\left(G_{\rho}\right)$ are roots of unity and, in particular, have absolute value 1 . Now, comparing (1.4) with (1.3) termwise, we obtain the following inequality.

Corollary 1.4. For any homomorphism $g: \pi=\pi_{1}(W) \rightarrow G$,

$$
\left|\operatorname{Hom}_{g}\left(\pi, G^{\prime}\right)\right| \leq|\operatorname{Hom}(\pi, \Gamma)| \text {. }
$$

This inequality is an equality if and only if $g(\pi) \subset G_{\rho}$ and $g^{*}\left(\zeta_{\rho}\right)([W])=1$ for all irreducible representations $\rho$ of $\Gamma$.

The inequality (1.7) does not hold for arbitrary groups $\pi$. For example, let $G^{\prime}=S_{3}$, the group of permutations of the set $\{1,2,3\}$, and let $q: G^{\prime} \rightarrow \mathbb{Z} / 2 \mathbb{Z}$ be the surjection sending all transpositions to $1(\bmod 2)$. Clearly, $\Gamma=\operatorname{Ker} q=\mathbb{Z} / 3 \mathbb{Z}$. Let $\pi$ be the group with $m \geq 1$ generators $x_{1}, \ldots, x_{m}$ and defining relations $x_{1}^{2}=x_{2}^{2}=\cdots=x_{m}^{2}$. A homomorphism $\pi \rightarrow \Gamma$ has to send all the generators $x_{1}, \ldots, x_{m}$ to the same element. Therefore, $\left|\operatorname{Hom}_{g}(\pi, \Gamma)\right|=3$. On the other hand, the homomorphism $\pi \rightarrow \mathbb{Z} / 2 \mathbb{Z}$ sending $x_{1}, \ldots, x_{m}$ to $1(\bmod 2)$ admits at least $3^{m}$ lifts to $G^{\prime}$ sending $x_{1}, \ldots, x_{m}$ to arbitrary transpositions.

A section of a group homomorphism $p: \pi^{\prime} \rightarrow \pi$ is a homomorphism $s: \pi \rightarrow \pi^{\prime}$ such that $p s=\mathrm{id}_{\pi}$. The set of sections of $p$ is denoted by $S_{*}(p)$.

Corollary 1.5. Let $p: \pi^{\prime} \rightarrow \pi=\pi_{1}(W)$ be a group epimorphism with finite kernel $\Phi$. Then

$$
\left|S_{*}(p)\right|=|\Phi| \sum_{\rho \in \operatorname{Irr}(\Phi), \pi_{\rho}=\pi}(|\Phi| / \operatorname{dim} \rho)^{-\chi(W)} \zeta_{\rho}([W]) .
$$

This is obtained from Theorem 1.1 by setting $G=\pi, G^{\prime}=\pi^{\prime}, \Gamma=\Phi, q=p$, and $g=\mathrm{id}: \pi \rightarrow \pi$. Corollary 1.4 implies that $\left|S_{*}(p)\right| \leq|\operatorname{Hom}(\pi, \Phi)|$. This inequality is an equality if and only if $\pi_{\rho}=\pi$ and $\zeta_{\rho}([W])=1$ for all irreducible representations $\rho$ of $\Phi$. The results of the next section imply that the number $\left|S_{*}(p)\right|$ is divisible by $|\Phi||Z(\Phi)|^{2 d-2}$, where $Z(\Phi)$ is the center of $\Phi$ and $d$ is the genus of $W$.

\section{The functions $\left\{v_{k}\right\}_{k}$}

The aim of this section is to deduce from Theorem 1.1 the following claim.

Theorem 2.1. Let $g: \pi=\pi_{1}(W) \rightarrow G$ be a group homomorphism. Then the number $\left|\operatorname{Hom}_{g}\left(\pi, G^{\prime}\right)\right|$ is divisible by $|\Gamma||Z(\Gamma)|^{2 d-2}$, where $Z(\Gamma)$ is the center of $\Gamma$ and $d$ is the genus of $W$.

Observe first that for each $k=1,2, \ldots$, the epimorphism $q: G^{\prime} \rightarrow G$ determines a function $v_{k}: H_{2}(G) \rightarrow \mathbb{C}$ by

$$
v_{k}(h)=\sum_{\rho \in \operatorname{Irr}(\Gamma), \operatorname{dim} \rho=k, G_{\rho}=G} \zeta_{\rho}(h) \in \mathbb{C},
$$


where $h \in H_{2}(G)$. It is well known that the dimension of any irreducible representation of $\Gamma$ divides $|\Gamma / Z(\Gamma)|$ and is smaller than or equal to $|\Gamma / Z(\Gamma)|^{1 / 2}$. Therefore $v_{k}=0$ if $k$ does not divide $|\Gamma / Z(\Gamma)|$ or $k>|\Gamma / Z(\Gamma)|^{1 / 2}$.

We can rewrite Formula (1.5) in terms of the functions $v_{1}, v_{2}, \ldots$ : for any epimorphism $g: \pi=\pi_{1}(W) \rightarrow G$,

$$
\left|\operatorname{Hom}_{g}\left(\pi, G^{\prime}\right)\right|=|\Gamma| \sum_{k \geq 1} v_{k}\left(g_{*}([W])\right)(|\Gamma| / k)^{-\chi(W)} .
$$

The next lemma summarizes the properties of the functions $\left\{v_{k}\right\}_{k}$.

Lemma 2.2. For $k=1,2, \ldots$, let $N_{k}$ be the number of equivalence classes of irreducible $k$-dimensional complex representations $\rho$ of $\Gamma$ such that $G_{\rho}=G$. Let $Q$ be the image of the homomorphism $q_{*}: H_{2}\left(G^{\prime}\right) \rightarrow H_{2}(G)$. Then

(a) For all $k$, the function $v_{k}$ takes only integer values and is zero outside $Q$. Moreover, all values of $v_{k}$ lie in the set $\left\{-N_{k},-N_{k}+1, \ldots, N_{k}-1, N_{k}\right\}$;

(b) For all $h \in Q$, we have $v_{1}(h)=N_{1}=\left|\Gamma /\left[\Gamma, G^{\prime}\right]\right|$;

(c) For all $k$, we have $v_{k}(0)=N_{k}$.

Proof. (a) It is well known that for any $h \in H_{2}(G)$ there are a closed connected oriented surface $\Sigma$ and a homomorphism $g: \pi_{1}(\Sigma) \rightarrow G$ such that $g_{*}([\Sigma])=h$. We say that the pair $(\Sigma, g)$ realizes $h$. Pick a representation $\rho$ of $\Gamma$ such that $G_{\rho} \neq G$. Adding to $\Sigma$ a handle and mapping its meridian to $1 \in G$ and its longitude to any element of $G-G_{\rho}$, we obtain a realization of $h$ by a surface $\Sigma^{\prime}$ and a homomorphism $\pi_{1}\left(\Sigma^{\prime}\right) \rightarrow G$ whose image meets $G-G_{\rho}$. Repeating this process, we can realize $h$ by a surface $\Sigma_{+}$with fundamental group $\pi$ and a homomorphism $g: \pi \rightarrow G$ such that $g(\pi) \subset G_{\rho}$ only when $G_{\rho}=G$. Formula (1.4) implies then that

$$
\left|\operatorname{Hom}_{g}\left(\pi, G^{\prime}\right)\right|=|\Gamma|^{2 d-1} \sum_{k \geq 1} v_{k}(h) k^{2-2 d},
$$

where $d$ is the genus of $\Sigma_{+}$. Any surface of bigger genus admits a degree one map to $\Sigma_{+}$. Such a map induces a surjection of fundamental groups. We can apply $(2.2)$ to the composition of this surjection with $g$. This implies that $\sum_{k \geq 1} v_{k}(h) k^{2-2 n} \in \mathbb{Q}$ for all $n \geq d$. By linear algebra, $v_{k}(h) \in \mathbb{Q}$ for all $k$.

By the remarks made before the statement of Corollary 1.4, for any irreducible representation $\rho$ of $\Gamma$ with $G_{\rho}=G$ and any $h \in H_{2}(G)$, the number $\zeta_{\rho}(h) \in \mathbb{C}$ is a root of unity. Thus, $v_{k}(h)$ is a sum of $N_{k}$ roots of unity. Hence, $v_{k}(h)$ is an algebraic integer. Therefore $v_{k}(h) \in \mathbb{Z}$ and $\left|v_{k}(h)\right| \leq N_{k}$.

If $h \notin Q$, then a homomorphism $g$ realizing $h$ as above cannot lift to $G^{\prime}$. Formula (2.2) and the argument after this formula show that $\sum_{k \geq 1} v_{k}(h) k^{2-2 n}=0$ for all sufficiently big natural numbers $n$. This gives a non-degenerate system of linear equations on $\left\{v_{k}(h)\right\}_{k}$. Therefore $v_{k}(h)=0$ for all $k$.

(b) By definition, $v_{1}(h)=\sum_{\rho} \zeta_{\rho}(h)$, where $\rho$ runs over all homomorphisms $\Gamma \rightarrow \mathbb{C}^{*}$ such that $G_{\rho}=G$. Formula (1.6) and the inclusion $h \in Q$ imply that $\zeta_{\rho}(h)=1$. Therefore $v_{1}(h)=N_{1}$ is simply the number of such $\rho$. The condition $G_{\rho}=G$ holds if and only if $\rho\left(a h a^{-1} h^{-1}\right)=1$ for all $a \in G^{\prime}, h \in \Gamma$. The latter holds if and only if $\rho\left(\left[\Gamma, G^{\prime}\right]\right)=1$. Thus, $N_{1}=\left|\Gamma /\left[\Gamma, G^{\prime}\right]\right|$.

(c) The equality $v_{k}(0)=N_{k}$ follows from the equality $\zeta_{\rho}(0)=1$ for all irreducible representations $\rho$ of $\Gamma$. 
As an exercise, the reader may verify that $v_{k}(-h)=v_{k}(h)$ and $v_{k}\left(h+k h^{\prime}\right)=v_{k}(h)$ for all $h \in H_{2}(G)$ and $h^{\prime} \in Q$.

Proof of Theorem 2.1. Replacing $G$ and $G^{\prime}$ by $g(\pi)$ and $q^{-1}(g(\pi))$, respectively, we can reduce ourselves to the case where $g$ is an epimorphism. In this case Theorem 2.1 follows from Formula (2.1), Lemma 2.2(a), and the fact that $v_{k}=0$ if $k$ does not divide $|\Gamma / Z(\Gamma)|=|\Gamma| /|Z(\Gamma)|$.

\section{The homological obstruction to lifting}

Consider in more detail the question of the existence of lifts to $G^{\prime}$ for a given homomorphism $g: \pi=\pi_{1}(W) \rightarrow G$. Replacing $G$ and $G^{\prime}$ by $g(\pi)$ and $q^{-1}(g(\pi))$, respectively, we can reduce ourselves to the case where $g$ is an epimorphism. Then Formula (2.1) computes $\left|\operatorname{Hom}_{g}\left(\pi, G^{\prime}\right)\right|$ in terms of the numbers $\left\{v_{k}(h)\right\}_{k}$, where $h=$ $g_{*}([W]) \in H_{2}(G)$. Thus, $g$ lifts to $G^{\prime}$ if and only if the right-hand side of (2.1) is non-zero.

Another approach to the same question stems from homological considerations. If an epimorphism $g: \pi \rightarrow G$ lifts to $G^{\prime}$, then the homology class $g_{*}([\Sigma]) \in H_{2}(G)$ necessarily lies in the image of the homomorphism $q_{*}: H_{2}\left(G^{\prime}\right) \rightarrow H_{2}(G)$. If the latter condition is satisfied, then we say that the homological obstruction to the lifting of $g$ to $G^{\prime}$ vanishes. In general, the vanishing of the homological obstruction may not imply that $g$ lifts to $G^{\prime}$. The next two theorems show that there are no further obstructions if the group $\Gamma=\operatorname{Ker} q$ is abelian or the genus of $W$ is big enough.

In the sequel, the symbol $\left[\Gamma, G^{\prime}\right]$ denotes the subgroup of $\Gamma$ generated by the commutators of elements of $\Gamma$ with elements of $G^{\prime}$.

Theorem 3.1. Suppose that the group $\Gamma$ is abelian. An epimorphism $g: \pi=$ $\pi_{1}(W) \rightarrow G$ lifts to $G^{\prime}$ if and only if the homological obstruction to the lifting vanishes. Moreover, if $g$ lifts to $G^{\prime}$, then

$$
\left|\operatorname{Hom}_{g}\left(\pi, G^{\prime}\right)\right|=|\Gamma|^{b}\left|\left[\Gamma, G^{\prime}\right]\right|^{-1},
$$

where $b=2-\chi(W)$ is the first Betti number of $W$.

Proof. Assume that the homological obstruction in question vanishes so that $h=$ $g_{*}([W]) \in \operatorname{Im} q_{*}$. By Lemma 2.2(b), we have $v_{1}(h)=\left|\Gamma /\left[\Gamma, G^{\prime}\right]\right|$. Since $\Gamma$ is abelian, all irreducible representations of $\Gamma$ are one-dimensional. So, $v_{k}(h)=0$ for $k \geq 2$. Now, the claim of the theorem directly follows from (2.1).

Formula (3.1) can be rewritten as

$$
\left|\operatorname{Hom}_{g}\left(\pi, G^{\prime}\right)\right|=|\operatorname{Hom}(\pi, \Gamma)| \times\left|\left[\Gamma, G^{\prime}\right]\right|^{-1} .
$$

This formula does not directly extend to groups $\pi$ distinct from the fundamental groups of closed oriented surfaces. For instance, if $\pi$ is a free group of rank $n$, then the left-hand and right-hand sides of (3.2) are equal respectively to $|\Gamma|^{n}$ and $|\Gamma|^{n}\left|\left[\Gamma, G^{\prime}\right]\right|^{-1}$. These numbers are equal if and only if $\left[\Gamma, G^{\prime}\right]=1$, i.e., if and only if $\Gamma$ lies in the center of $G^{\prime}$.

Theorem 3.2. Suppose that the first Betti number $b=2-\chi(W)$ of $W$ satisfies

$$
b>\log _{2}\left(\left|\left[\Gamma, G^{\prime}\right]\right|-1\right) \text {. }
$$


An epimorphism $g: \pi=\pi_{1}(W) \rightarrow G$ lifts to $G^{\prime}$ if and only if the homological obstruction to the lifting vanishes. Moreover, if $g$ lifts to $G^{\prime}$, then

$$
\left|\operatorname{Hom}_{g}\left(\pi, G^{\prime}\right)\right| \geq|\Gamma|^{b}\left|\left[\Gamma, G^{\prime}\right]\right|^{-1} \times\left(1-\frac{\left|\left[\Gamma, G^{\prime}\right]\right|-1}{2^{b}}\right) .
$$

It is understood that if $\left[\Gamma, G^{\prime}\right]=1$, then the condition (3.3) is empty.

Proof. For $k=1,2, \ldots$, denote by $M_{k}$ the number of equivalence classes of irreducible $k$-dimensional representations of $\Gamma$. It is clear that $\sum_{k>1} M_{k} k^{2}=|\Gamma|$.

Pick any $h \in H_{2}(G)$. Let $N_{k}$ be the same number as in Lemma 2.2. The inequality $\left|v_{k}(h)\right| \leq N_{k}$ established in Lemma 2.2 and the obvious inequality $N_{k} \leq M_{k}$ imply that for any integer $n<2$,

$$
\begin{gathered}
\sum_{k \geq 1} v_{k}(h) k^{n} \geq v_{1}(h)-\sum_{k \geq 2}\left|v_{k}(h)\right| k^{n} \geq v_{1}(h)-\sum_{k \geq 2} M_{k} k^{n} \\
=v_{1}(h)-\sum_{k \geq 2} M_{k} k^{2} k^{n-2} \geq v_{1}(h)-\left(\sum_{k \geq 2} M_{k} k^{2}\right) 2^{n-2} \\
=v_{1}(h)-2^{n-2}\left(|\Gamma|-M_{1}\right) \geq v_{1}(h)-2^{n-2}\left(|\Gamma|-N_{1}\right) .
\end{gathered}
$$

If $h \in \operatorname{Im} q_{*}$, then $v_{1}(h)=N_{1}=\left|\Gamma /\left[\Gamma, G^{\prime}\right]\right|$ by Lemma 2.2. This gives

$$
\sum_{k \geq 1} v_{k}(h) k^{n} \geq\left|\Gamma /\left[\Gamma, G^{\prime}\right]\right|\left(1-\frac{\left|\left[\Gamma, G^{\prime}\right]\right|-1}{2^{2-n}}\right) .
$$

Setting $n=\chi(W)=2-b$ and combining with (2.1), we obtain (3.4).

\section{Geometric applications of Theorem 1.1}

Enumeration of principal fiber bundles. Let $\mathcal{P}=\mathcal{P}(W, \Gamma)$ be the set of isomorphism classes of principal $\Gamma$-bundles over $W$. Recall that a homomorphism $g: \pi \rightarrow \Gamma$ determines a principal $\Gamma$-bundle $\xi_{g}$ over $W$, and every principal $\Gamma$-bundle over $W$ is isomorphic to $\xi_{g}$ for some $g$. Two homomorphisms $g_{1}, g_{2}: \pi \rightarrow \Gamma$ determine isomorphic principal $\Gamma$-bundles over $W$ if and only if $g_{1}=h g_{2} h^{-1}$ for some $h \in \Gamma$. Therefore $\mathcal{P}=\operatorname{Hom}(\pi, \Gamma) / \Gamma$, where $\Gamma$ acts on $\operatorname{Hom}(\pi, \Gamma)$ by conjugation. The stabilizer of $g \in \operatorname{Hom}(\pi, \Gamma)$ under this action is the group $\left\{h \in \Gamma \mid h g h^{-1}=g\right\}$ isomorphic to the group of automorphisms $\operatorname{Aut}\left(\xi_{g}\right)$ of $\xi_{g}$. Combining these facts, we obtain

$$
|\operatorname{Hom}(\pi, \Gamma)|=\sum_{\xi \in \mathcal{P}} \frac{|\Gamma|}{|\operatorname{Aut}(\xi)|} .
$$

The Frobenius-Mednykh formula (1.3) implies therefore that

$$
\sum_{\xi \in \mathcal{P}(W ; \Gamma)} \frac{1}{|\operatorname{Aut}(\xi)|}=\sum_{\rho \in \operatorname{Irr}(\Gamma)}\left(\frac{|\Gamma|}{\operatorname{dim} \rho}\right)^{-\chi(W)} .
$$

Theorem 1.1 yields a relative version of (4.1) as follows. Fix a principal $G$-bundle $\xi$ over $W$. By a lift of $\xi$ to a principal $G^{\prime}$-bundle, we mean a pair (a principal $G^{\prime}$-bundle $\xi^{\prime}$ over $W$, an isomorphism of $G$-bundles $\left.f: \xi^{\prime} / \Gamma \cong \xi\right)$. Here $\xi^{\prime} / \Gamma$ is the principal $G$-bundle over $W$ obtained by factorizing the total space of $\xi^{\prime}$ by $\Gamma$. An isomorphism $\left(\xi_{1}^{\prime}, f_{1}\right) \approx\left(\xi_{2}^{\prime}, f_{2}\right)$ of two such lifts of $\xi$ is an isomorphism of principal $G^{\prime}$-bundles $\xi_{1}^{\prime} \rightarrow \xi_{2}^{\prime}$ such that the induced isomorphism of principal $G$-bundles $\xi_{1}^{\prime} / \Gamma \rightarrow \xi_{2}^{\prime} / \Gamma$ 
composed with $f_{2}: \xi_{2}^{\prime} / \Gamma \rightarrow \xi$ gives $f_{1}$. In particular, an automorphism of a lift $\left(\xi^{\prime}, f\right)$ of $\xi$ is an automorphism of $\xi^{\prime}$ inducing the identity on $\xi^{\prime} / \Gamma$. Such automorphisms form a group denoted by $\operatorname{Aut}_{\xi}\left(\xi^{\prime}\right)$. The set of isomorphism classes of lifts of $\xi$ to principal $G^{\prime}$-bundles is denoted by $\mathcal{P}(\xi)$.

Fix a homomorphism $g: \pi \rightarrow G$ such that $\xi=\xi_{g}$. It is clear that every homomorphism $g^{\prime} \in \operatorname{Hom}_{g}\left(\pi, G^{\prime}\right)$ determines a lift of $\xi$ to a principal $G^{\prime}$-bundle, and every such lift arises from some $g^{\prime} \in \operatorname{Hom}_{g}\left(\pi, G^{\prime}\right)$. Two homomorphisms $g_{1}^{\prime}, g_{2}^{\prime} \in \operatorname{Hom}_{g}\left(\pi, G^{\prime}\right)$ determine isomorphic lifts of $\xi$ if and only if $g_{1}^{\prime}=h g_{2}^{\prime} h^{-1}$ for some $h \in \Gamma$. Therefore

$$
\mathcal{P}(\xi)=\operatorname{Hom}_{g}\left(\pi, G^{\prime}\right) / \Gamma,
$$

where $\Gamma$ acts on $\operatorname{Hom}_{g}\left(\pi, G^{\prime}\right)$ by conjugation. The stabilizer of any homomorphism $g^{\prime} \in \operatorname{Hom}_{g}\left(\pi, G^{\prime}\right)$ under this action is the group $\left\{h \in \Gamma \mid h g^{\prime} h^{-1}=g^{\prime}\right\}$ isomorphic to $\operatorname{Aut}_{\xi}\left(\xi_{g^{\prime}}\right)$. Combining these facts, we obtain

$$
\left|\operatorname{Hom}_{g}\left(\pi, G^{\prime}\right)\right|=\sum_{\xi^{\prime} \in \mathcal{P}(\xi)} \frac{|\Gamma|}{\left|\operatorname{Aut}_{\xi}\left(\xi^{\prime}\right)\right|} .
$$

Theorem 1.1 implies that

$$
\sum_{\xi^{\prime} \in \mathcal{P}(\xi)} \frac{1}{\left|\operatorname{Aut}_{\xi}\left(\xi^{\prime}\right)\right|}=\sum_{\rho \in \operatorname{Irr}(\Gamma), G_{\rho} \supset g(\pi)}\left(\frac{|\Gamma|}{\operatorname{dim} \rho}\right)^{-\chi(W)} g^{*}\left(\zeta_{\rho}\right)([W]) .
$$

Formula (4.2) and Theorem 2.1 imply that $\sum_{\xi^{\prime} \in \mathcal{P}(\xi)} 1 /\left|\operatorname{Aut}_{\xi}\left(\xi^{\prime}\right)\right|$ is an integer divisible by $|Z(\Gamma)|^{2 d-2}$, where $d$ is the genus of $W$. Corollary 1.4 gives

$$
\sum_{\xi^{\prime} \in \mathcal{P}(\xi)} 1 /\left|\operatorname{Aut}_{\xi}\left(\xi^{\prime}\right)\right| \leq|\Gamma|^{-1}|\operatorname{Hom}(\pi, \Gamma)|
$$

Formula (4.3) implies that $\xi$ lifts to a principal $G^{\prime}$-bundle if and only if the right hand side of (4.3) is non-zero. Theorems 3.1 and 3.2 show that in the case where $\Gamma$ is abelian or the genus of $W$ is big enough, $\xi$ lifts to a principal $G^{\prime}$-bundle if and only if the homology class $g_{*}([W]) \in H_{2}(g(\pi))$ lies in the image of the homomorphism $q_{*}: H_{2}\left(q^{-1}(g(\pi))\right) \rightarrow H_{2}(g(\pi))$.

Enumeration of sections. Theorem 1.1 may be used to count homotopy classes of sections of locally trivial fiber bundles over the surface $W$. Let $p: E \rightarrow W$ be a locally trivial fiber bundle with fiber $F$. A section of $p$ is a continuous mapping $s: W \rightarrow E$ such that $p s=\mathrm{id}_{W}$. Two sections of $p$ are homotopic if they can be deformed into each other in the class of sections of $p$. We say that two sections $W \rightarrow E$ are obtained from each other by bubbling if they coincide on the complement of a small open disc $D \subset W$. The restrictions of such two sections on the closed disc $\bar{D} \subset W$ form then a mapping $S^{2} \rightarrow E$, a "bubble". Two sections of $p$ are bubble equivalent if they may be obtained from each other by a finite sequence of bubblings. Decomposing a deformation of a section into local deformations, one easily observes that homotopic sections are bubble equivalent. (If $\pi_{2}(F)=0$, then the converse is also true so that the bubble equivalence is just the homotopy.) Denote the set of bubble equivalence classes of sections of $p$ by $\mathcal{S}(p)$. We shall count the elements of this set with certain weights, see Formula (4.6) below.

The definition of $\mathcal{S}(p)$ has a pointed version as follows. Fix a base point $e \in E$ and set $w=p(e) \in W$. A section $s: W \rightarrow E$ of $p$ is pointed if $s(w)=e$. Two pointed 
sections of $p$ are homotopic if they can be deformed into each other in the class of pointed sections of $p$. The definitions of the bubbling and the bubbling equivalence extend to pointed sections in the obvious way with the only difference that the disk $D$ in the definition of a bubbling should lie in $W-\{w\}$. As above, homotopic pointed sections are bubble equivalent, and the converse is true if $\pi_{2}(F)=0$. We denote the set of bubble equivalence classes of pointed sections of $p$ by $\mathcal{S}_{*}(p)$.

Suppose from now on that the fiber $F=p^{-1}(w)$ of $p$ is path-connected. Set $\pi=\pi_{1}(W, w), \pi^{\prime}=\pi_{1}(E, e)$, and $\Phi=\pi_{1}(F, e)$. The exact homotopy sequence of $p$ shows that the homomorphism $p_{\#}: \pi^{\prime} \rightarrow \pi$ is surjective and $\operatorname{Ker} p_{\#}=\Phi$. By Section 1, every irreducible representation $\rho$ of $\Phi$ determines a subgroup $\pi_{\rho}$ of $\pi$ and a cohomology class $\zeta_{\rho} \in H^{2}\left(\pi_{\rho} ; \mathbb{C}^{*}\right)$.

For any pointed section $s: W \rightarrow E$ of $p$, the induced homomorphism $s_{\#}: \pi \rightarrow \pi^{\prime}$ is a section of $p_{\#}$. It is clear that $s_{\#}$ is preserved under the bubblings of $s$. It is easy to check that the resulting mapping

$$
\mathcal{S}_{*}(p) \rightarrow S_{*}\left(p_{\#}\right), \quad s \mapsto s_{\#}
$$

is a bijection. Thus, $\left|\mathcal{S}_{*}(p)\right|=\left|S_{*}(p)\right|$. Corollary 1.5 implies the following claim.

Theorem 4.1. If $\Phi$ is finite, then

$$
\left|\mathcal{S}_{*}(p)\right|=|\Phi| \sum_{\rho \in \operatorname{Irr}(\Phi), \pi_{\rho}=\pi}(|\Phi| / \operatorname{dim} \rho)^{-\chi(W)} \zeta_{\rho}([W]) .
$$

We now rewrite (4.5) in terms of non-pointed sections of $p$. Since $F$ is pathconnected, any section of $p$ is homotopic to a pointed section. This shows that the natural mapping $\mathcal{S}_{*}(p) \rightarrow \mathcal{S}(p)$ is surjective. This mapping may be described in terms of an action of $\Phi$ on $\mathcal{S}_{*}(p)$ as follows. The group $\Phi$ acts on the set $S_{*}\left(p_{\#}\right)$ by conjugation. This defines an action of $\Phi$ on $\mathcal{S}_{*}(p)$ via the bijection (4.4). It is easy to see that the orbits of the latter action are precisely the preimages of elements of $\mathcal{S}(p)$ under the natural mapping $\mathcal{S}_{*}(p) \rightarrow \mathcal{S}(p)$. Thus, $\mathcal{S}(p)=\mathcal{S}_{*}(p) / \Phi$. For $s \in \mathcal{S}(p)$, let $\operatorname{Aut}(s) \subset \Phi$ be the stabilizer of an element of $\mathcal{S}_{*}(p)$ projecting to $s$. The group $\operatorname{Aut}(s)$ is well defined up to conjugation in $\Phi$. If $\Phi$ is finite, then

$$
\left|\mathcal{S}_{*}(p)\right|=\sum_{s \in \mathcal{S}(p)} \frac{|\Phi|}{|\operatorname{Aut}(s)|} .
$$

Formula (4.5) may now be rewritten as

$$
\sum_{s \in \mathcal{S}(p)} \frac{1}{|\operatorname{Aut}(s)|}=\sum_{\rho \in \operatorname{Irr}(\Phi), \pi_{\rho}=\pi}(|\Phi| / \operatorname{dim} \rho)^{-\chi(W)} \zeta_{\rho}([W]) .
$$

Theorem 2.1 and Corollary 1.4 imply that $\sum_{s \in \mathcal{S}(p)} 1 /|\operatorname{Aut}(s)|$ is an integer divisible by $|Z(\Phi)|^{2 d-2}$, where $d$ is the genus of $W$, and

$$
\sum_{s \in \mathcal{S}(p)} 1 /|\operatorname{Aut}(s)| \leq|\Phi|^{-1}|\operatorname{Hom}(\pi, \Phi)| .
$$

Corollary 4.2. Under the assumptions of Theorem 4.1 the bundle $p: E \rightarrow W$ has a section if and only if

$$
\sum_{\rho \in \operatorname{Irr}(\Phi), \pi_{\rho}=\pi}(\operatorname{dim} \rho)^{\chi(W)} \zeta_{\rho}([W]) \neq 0 .
$$


Note that the left-hand side of this formula is a non-negative rational number for all $p$, as it follows from Theorem 4.1. In the case where $\Phi$ is abelian or the genus of $W$ is bigger than $(1 / 2) \log _{2}\left(\left|\left[\Phi, \pi^{\prime}\right]\right|-1\right)$, Theorems 3.1 and 3.2 imply that the bundle $p$ has a section if and only if the induced homomorphism $p_{*}: H_{2}(E) \rightarrow H_{2}(W)$ is surjective.

In the case of a trivial fiber bundle, Theorem 4.1 amounts to computing the number of pointed homotopy classes of maps $W \rightarrow F$. In this case, all the cohomology classes $\zeta_{\rho}$ are trivial and Theorem 4.1 follows from the equality $\left|\mathcal{S}_{*}(p)\right|=\left|S_{*}(p)\right|$ and the Frobenius-Mednykh formula (1.3).

Enumeration of lifts of maps. Given an arbitrary locally trivial fiber bundle $p$ : $E \rightarrow X$ and a map $f$ from the surface $W$ to $X$, one may be interested in counting the number of homotopy classes of lifts of $f$ to $E$. By a lift of $f$ to $E$, we mean a mapping $f^{\prime}: W \rightarrow E$ such that $p f^{\prime}=f$. For $X=W$ and $f=\operatorname{id}_{W}$, we recover the setting of the previous subsection. All definitions and results given there extend to arbitrary $p, f$ with the obvious changes. The key observation is that the lifts of $f$ to $E$ bijectively correspond to the sections of the induced fiber bundle $f^{*}(p)$ over $W$.

Non-abelian cohomology of surfaces. Theorem 1.1 yields interesting information about 1-dimensional non-abelian cohomology of the fundamental groups of surfaces. We begin by recalling the definition of the 1-dimensional non-abelian cohomology of an arbitrary group $\pi$, cf. [Se]. Fix a left action of $\pi$ on a group $\Phi$, i.e., a homomorphism $\pi \rightarrow$ Aut $\Phi$. A map $\alpha: \pi \rightarrow \Phi$ is a cocycle if $\alpha(a b)=\alpha(a) a(\alpha(b))$ for all $a, b \in \pi$. Here $a(\alpha(b)) \in \Phi$ is obtained by the action of $a$ on $\alpha(b)$. For example, the mapping $\pi \rightarrow\{1\} \subset \Phi$ is a cocycle. The set of all cocycles $\pi \rightarrow \Phi$ is denoted by $Z^{1}(\pi ; \Phi)$. The group $\Phi$ acts on $Z^{1}(\pi ; \Phi)$ by

$$
(\varphi \alpha)(a)=\varphi \alpha(a)(a \varphi)^{-1}
$$

for all $\varphi \in \Phi, \alpha \in Z^{1}(\pi ; \Phi)$, and $a \in \pi$. The quotient set of this action is denoted by $H^{1}(\pi ; \Phi)$ and called the (nonabelian) cohomology of $\pi$ with coefficients in $\Phi$. For $h \in H^{1}(\pi ; \Phi)$, let $\operatorname{Aut}(h) \subset \Phi$ be the stabilizer of any cocycle representing $h$. The group $\operatorname{Aut}(h)$ is well defined up to conjugation in $\Phi$.

If $\pi$ is finitely generated and $\Phi$ is finite, then both sets $Z^{1}(\pi ; \Phi)$ and $H^{1}(\pi ; \Phi)$ are finite. Put

$$
\mathcal{M}(\pi ; \Phi)=\sum_{h \in H^{1}(\pi ; \Phi)} \frac{1}{|\operatorname{Aut}(h)|} \in \mathbb{Q} .
$$

We view $\mathcal{M}(\pi ; \Phi)$ as the global measure of the set $H^{1}(\pi ; \Phi)$ counting its elements with the weights $1 / \mid$ Aut|. Since any $h \in H^{1}(\pi ; \Phi)$ can be represented by precisely $|\Phi| /|\operatorname{Aut}(h)|$ cocycles,

$$
\mathcal{M}(\pi ; \Phi)=|\Phi|^{-1}\left|Z^{1}(\pi ; \Phi)\right|
$$

The definitions of Section 1 can be adapted to this setting as follows. With an irreducible representation $\rho: \Phi \rightarrow G L_{n}(\mathbb{C})$ of $\Phi$ we associate the group $\pi_{\rho} \subset \pi$ consisting of all $a \in \pi$ such that the representation $\varphi \mapsto \rho\left(a^{-1} \varphi\right)$ of $\Phi$ is equivalent to $\rho$. This means that there is a matrix $M_{a} \in G L_{n}(\mathbb{C})$ such that $\rho\left(a^{-1} \varphi\right)=M_{a}^{-1} \rho(\varphi) M_{a}$ for all $\varphi \in \Phi$. Then there is a family of non-zero complex numbers $\left\{\zeta_{a, b}\right\}_{a, b \in \pi_{\rho}}$ such that $\zeta_{a, b} M_{a} M_{b}=M_{a b}$ for all $a, b \in \pi_{\rho}$. This family is a 2-cocycle representing a well-defined cohomology class $\zeta_{\rho} \in H^{2}\left(\pi_{\rho} ; \mathbb{C}^{*}\right)$. 
Theorem 4.3. For any action of $\pi=\pi_{1}(W)$ on a finite group $\Phi$,

$$
\mathcal{M}(\pi ; \Phi)=\sum_{\rho \in \operatorname{Irr}(\Phi), \pi_{\rho}=\pi}(|\Phi| / \operatorname{dim} \rho)^{-\chi(W)} \zeta_{\rho}([W]) .
$$

Proof. Let $\pi^{\prime}$ be the set of pairs $(\varphi \in \Phi, a \in \pi)$ with multiplication $(\varphi, a)\left(\varphi^{\prime}, a^{\prime}\right)=$ $\left(\varphi\left(a \varphi^{\prime}\right), a a^{\prime}\right)$. It is easy to check that $\pi^{\prime}$ is a group. The formula $p(\varphi, a)=a$ defines an epimorphism $p: \pi^{\prime} \rightarrow \pi$ with kernel $\{(\varphi, 1)\}_{\varphi \in \Phi}=\Phi$. Every cocycle $\alpha: \pi \rightarrow \Phi$ defines a section $s_{\alpha}$ of $p$ by $s_{\alpha}(a)=(\alpha(a), a)$ for $a \in \pi$. The formula $\alpha \mapsto s_{\alpha}$ establishes a bijection between the set $Z^{1}(\pi ; \Phi)$ and the set $S_{*}(p)$ of the sections of $p$. Therefore

$$
\mathcal{M}(\pi ; \Phi)=|\Phi|^{-1}\left|Z^{1}(\pi ; \Phi)\right|=|\Phi|^{-1}\left|S_{*}(p)\right| .
$$

It remains to apply Corollary 1.5 and to observe that the definitions of $\pi_{\rho}$ and $\zeta_{\rho}$ given in Section 1 are equivalent in the present setting to the definitions given before the statement of the theorem. (The key point is that every $a \in \pi$ has a canonical lift $(1, a)$ to $\pi^{\prime}$ and

$$
(1, a)^{-1}(\varphi, 1)(1, a)=\left(a^{-1} \varphi, 1\right)
$$

for all $\varphi \in \Phi$.

Formula (4.7) and the remarks after Corollary 1.5 imply that $\mathcal{M}(\pi ; \Phi)$ is an integer divisible by $|Z(\Phi)|^{2 d-2}$, where $d$ is the genus of $W$, and

$$
\mathcal{M}(\pi ; \Phi) \leq|\Phi|^{-1}|\operatorname{Hom}(\pi, \Phi)| .
$$

\section{Miscellaneous algebra}

We discuss miscellaneous algebraic notions and results related to Theorem 1.1.

Extremal homology classes. We call a homology class $h \in H_{2}(G)$ extremal (with respect to the given epimorphism $\left.q: G^{\prime} \rightarrow G\right)$ if $\zeta_{\rho}(h)=1$ for all irreducible representations $\rho$ of $\Gamma=\operatorname{Ker} q$ such that $G_{\rho}=G$. For example, the zero homology class $h=0$ is extremal. It is clear that the extremal homology classes form a subgroup of $H_{2}(G)$.

For each $k \geq 1$, the function $v_{k}$ introduced in Section 2 takes on all extremal classes the same value which is the maximal value of $v_{k}$. In particular, if $h \in H_{2}(G)$ is extremal, then $v_{1}(h)=v_{1}(0)>0$. By Lemma 2.2(a), all extremal homology classes lie in $Q=\operatorname{Im}\left(q_{*}: H_{2}\left(G^{\prime}\right) \rightarrow H_{2}(G)\right)$. Let $a \in \mathbb{Z}$ be the least common multiple of the numbers $\operatorname{dim} \rho$, where $\rho$ runs over all irreducible representations of $\Gamma$. The properties of $\zeta_{\rho}$ imply that all elements of the group $a Q \subset Q$ are extremal.

Quasi-epimorphisms. A homomorphism $g: \pi \rightarrow G$ is a quasi-epimorphism (with respect to $\left.q: G^{\prime} \rightarrow G\right)$ if

$$
g(\pi) \cap\left(G-G_{\rho}\right) \neq \emptyset
$$

for all irreducible representations $\rho$ of $\Gamma$ such that $G_{\rho} \neq G$. In particular, all epimorphisms $\pi \rightarrow G$ are quasi-epimorphisms. If $G_{\rho}=G$ for all $\rho$, then all homomorphisms $\pi \rightarrow G$ are quasi-epimorphisms. 
For a quasi-epimorphism $g: \pi=\pi_{1}(W) \rightarrow G$, Theorem 1.1 yields the same formula (1.5) as for an epimorphism. Therefore

$$
\left|\operatorname{Hom}_{g}\left(\pi, G^{\prime}\right)\right| \leq|\Gamma| \sum_{\rho \in \operatorname{Irr}(\Gamma), G_{\rho}=G}(|\Gamma| / \operatorname{dim} \rho)^{-\chi(W)} .
$$

This inequality is an equality if and only if $g_{*}([W]) \in H_{2}(G)$ is an extremal homology class.

The genus norm. As was already mentioned above, for any non-zero $h \in H_{2}(G)$, there are a closed connected oriented surface $\Sigma$ of positive genus and a homomorphism $g: \pi_{1}(\Sigma) \rightarrow G$ such that $g_{*}([\Sigma])=h$. Let $|h| \geq 1$ be the minimal genus of such $\Sigma$. By definition, $|0|=0$. Clearly, $\left|h+h^{\prime}\right| \leq|h|+\left|h^{\prime}\right|$ for all $h, h^{\prime} \in H_{2}(G)$. Also, $|-h|=|h|$ and $|h|=0$ if and only if $h=0$. We call the mapping $H_{2}(G) \rightarrow \mathbb{Z}, h \mapsto|h|$ the genus norm.

The functions $v_{1}, v_{2}, \ldots$ from Section 2 may help to estimate the genus norm as follows. Define a mapping $v: H_{2}(G) \times \mathbb{Z} \rightarrow \mathbb{Q}$ by

$$
v(h, n)=\sum_{k \geq 1} v_{k}(h) k^{-2 n},
$$

where $h \in H_{2}(G)$ and $n \in \mathbb{Z}$.

Lemma 5.1. If $G_{\rho}=G$ for all irreducible representations $\rho$ of $\Gamma$, then $v(h, n) \geq 0$ for all $h \in H_{2}(G)$ and all $n \geq|h|-1$.

Proof. If $h=0$, then $v(h, n)>0$ for all $n \in \mathbb{Z}$, as directly follows from Lemma 2.2(c). Suppose that $h \neq 0$. Let $\Sigma$ be a closed connected oriented surface of genus $|h| \geq 1$ and let $g: \pi_{1}(\Sigma) \rightarrow G$ be a homomorphism such that $g_{*}([\Sigma])=h$. Theorem 1.1 and the assumption $G_{\rho}=G$ for all $\rho$ imply that

$$
\left|\operatorname{Hom}_{g}\left(\pi_{1}(\Sigma), G^{\prime}\right)\right|=|\Gamma|^{1-\chi(\Sigma)} v\left(h,-\frac{\chi(\Sigma)}{2}\right)=|\Gamma|^{2|h|-1} v(h,|h|-1) .
$$

Hence, $v(h,|h|-1) \geq 0$. Similarly $v(h, n) \geq 0$ for all $n \geq|h|$, cf. the argument after $(2.2)$.

For $h \in H_{2}(G)$, denote by $\langle h, q\rangle$ the minimal non-negative integer such that $v(h, n) \geq 0$ for all $n \geq\langle h, q\rangle$. Lemma 5.1 implies that such an integer exists and $|h| \geq\langle h, q\rangle+1$. Varying $q$ in the class of group epimorphisms with target $G$ and finite kernel satisfying the conditions of Lemma 5.1, we obtain a family of estimates from below for the genus norm on $H_{2}(G)$. The author does not know whether these estimates may be non-trivial. Explicit examples and computations would be welcome.

\section{A generalization of Theorem 1.1}

Theorem 1.1 admits a generalization in which the lifts of $g$ to $G^{\prime}$ are counted with weights determined by a 2 -cocycle on $G^{\prime}$. To state this generalization, we first extend the definition of $\zeta_{\rho}$ to projective representations of $\Gamma$.

Fix throughout this section a 2-cocycle $\theta=\left\{\theta_{a, b} \in \mathbb{C}^{*}\right\}_{a, b \in G^{\prime}}$ on $G^{\prime}$. A mapping $\rho: \Gamma \rightarrow G L_{n}(\mathbb{C})$ with $n=1,2, \ldots$ is a $\theta$-representation of $\Gamma$ if $\rho(1)$ is the unit $n \times n$ matrix and $\rho(g) \rho(h)=\theta_{g, h} \rho(g h)$ for all $g, h \in \Gamma$. For any matrix $M \in G L_{n}(\mathbb{C})$ and a $\theta$-representation $\rho: \Gamma \rightarrow G L_{n}(\mathbb{C})$, the mapping $M^{-1} \rho M: \Gamma \rightarrow G L_{n}(\mathbb{C})$ 
sending $h \in \Gamma$ to $M^{-1} \rho(h) M$, is a $\theta$-representation. We say that two $\theta$-representations $\rho: \Gamma \rightarrow G L_{n}(\mathbb{C})$ and $\rho^{\prime}: \Gamma \rightarrow G L_{n^{\prime}}(\mathbb{C})$ are equivalent and write $\rho \sim \rho^{\prime}$ if $n=n^{\prime}$ and $\rho^{\prime}=M^{-1} \rho M$ for some $M \in G L_{n}(\mathbb{C})$. Clearly, $\sim$ is an equivalence relation on the set of $\theta$-representations of $\Gamma$. Denote by $\mathcal{R}_{\theta}$ the corresponding set of equivalence classes.

The cocycle $\theta$ determines an action of $G^{\prime}$ on $\mathcal{R}_{\theta}$ as follows. Given $a \in G^{\prime}$ and a $\theta$-representation $\rho: \Gamma \rightarrow G L_{n}(\mathbb{C})$, consider the mapping $a \rho: \Gamma \rightarrow G L_{n}(\mathbb{C})$, whose value on any $h \in \Gamma$ is given by

$$
a \rho(h)=\frac{\theta_{a^{-1}, h a} \theta_{h, a}}{\theta_{a, a^{-1}} \theta_{1,1}} \rho\left(a^{-1} h a\right) .
$$

Lemma 6.1. The mapping a $\rho$ is a $\theta$-representation of $\Gamma$. The formula $(a, \rho) \mapsto a \rho$ defines a left action of $G^{\prime}$ on $\mathcal{R}_{\theta}$. This action induces a left action of $G$ on $\mathcal{R}_{\theta}$.

Given a $\theta$-representation $\rho$ of $\Gamma$, denote by $G_{\rho}$ be the stabilizer of $\rho$, i.e., the subgroup of $G$ consisting of all $\alpha \in G$ such that $a \rho \sim \rho$ for some (and then for all) $a \in q^{-1}(\alpha) \subset G^{\prime}$. The group $G_{\rho}$ depends only on the equivalence class of $\rho$.

Let $\rho: \Gamma \rightarrow G L_{n}(\mathbb{C})$ be a $\theta$-representation of $\Gamma$, which is irreducible in the sense that the only linear subspaces of $\mathbb{C}^{n}$ preserved under the induced projective action of $\Gamma$ are 0 and $\mathbb{C}^{n}$. We define a cohomology class $\zeta_{\rho} \in H^{2}\left(G_{\rho} ; \mathbb{C}^{*}\right)$. Fix for each $\alpha \in G_{\rho}$, an element $\widetilde{\alpha}$ of $q^{-1}(\alpha)$. By definition of $G_{\rho}$, for $\alpha \in G_{\rho}$, there is a matrix $M_{\alpha} \in G L_{n}(\mathbb{C})$ such that $\widetilde{\alpha} \rho=M_{\alpha}^{-1} \rho M_{\alpha}$. The irreducibility of $\rho$ implies that $M_{\alpha}$ is unique up to multiplication by an element of $\mathbb{C}^{*}$; we fix $M_{\alpha}$ for all $\alpha$.

Lemma 6.2. For any $\alpha, \beta \in G_{\rho}$, there is a unique $\zeta_{\alpha, \beta} \in \mathbb{C}^{*}$ such that

$$
\zeta_{\alpha, \beta} M_{\alpha} M_{\beta}=\theta_{\widetilde{\alpha}, \widetilde{\beta}} \theta_{\widetilde{\alpha \beta}, \widetilde{\alpha \beta}^{-1} \widetilde{\alpha} \widetilde{\beta}}^{-1} M_{\alpha \beta} \rho\left(\widetilde{\alpha \beta}^{-1} \widetilde{\alpha} \widetilde{\beta}\right) .
$$

The family $\left\{\zeta_{\alpha, \beta}\right\}_{\alpha, \beta}$ is a 2-cocycle on $G_{\rho}$. Its cohomology class $\zeta_{\rho} \in H^{2}\left(G_{\rho} ; \mathbb{C}^{*}\right)$ depends only on the equivalence class of $\rho$ and does not depend on the choice of the matrices $\left\{M_{\alpha}\right\}_{\alpha}$ or the lifts $\{\widetilde{\alpha}\}_{\alpha}$.

There is a relationship between $\zeta_{\rho}$ and the cohomology class of the cocycle $\theta$. Consider the group $G_{\rho}^{\prime}=q^{-1}\left(G_{\rho}\right) \subset G^{\prime}$ and let $[\theta]_{\rho} \in H^{2}\left(G_{\rho}^{\prime} ; \mathbb{C}^{*}\right)$ denote the cohomology class of the restriction of $\theta$ to $G_{\rho}^{\prime}$. In generalization of (1.6), we have

$$
(\operatorname{dim} \rho) q^{*}\left(\zeta_{\rho}\right)=(\operatorname{dim} \rho)[\theta]_{\rho}
$$

where $q^{*}: H^{2}\left(G_{\rho} ; \mathbb{C}^{*}\right) \rightarrow H^{2}\left(G_{\rho}^{\prime} ; \mathbb{C}^{*}\right)$ is the homomorphism induced by $q$.

Theorem 6.3. Let $\theta=\left\{\theta_{a, b} \in \mathbb{C}^{*}\right\}_{a, b \in G^{\prime}}$ be a 2-cocycle on $G^{\prime}$ representing a cohomology class $[\theta] \in H^{2}\left(G^{\prime} ; \mathbb{C}^{*}\right)$. For any group homomorphism $g: \pi=\pi_{1}(W) \rightarrow G$,

$$
\sum_{g^{\prime} \in \operatorname{Hom}_{g}\left(\pi, G^{\prime}\right)}\left(g^{\prime}\right)^{*}([\theta])([W])=|\Gamma| \sum_{\rho, G_{\rho} \supset g(\pi)}(|\Gamma| / \operatorname{dim} \rho)^{-\chi(W)}\left(g^{*}\left(\zeta_{\rho}\right)([W])\right)^{-1},
$$

where $\rho$ ranges over the equivalence classes of irreducible $\theta$-representations of $\Gamma$ such that $G_{\rho} \supset g(\pi)$.

Here $\left(g^{\prime}\right)^{*}([\theta])([W]) \in \mathbb{C}^{*}$ and $g^{*}\left(\zeta_{\rho}\right)([W]) \in \mathbb{C}^{*}$ are the evaluations of $\left(g^{\prime}\right)^{*}([\theta])$, $g^{*}\left(\zeta_{\rho}\right) \in H^{2}\left(\pi ; \mathbb{C}^{*}\right)$ on $[W] \in H_{2}(W)=H_{2}(\pi)$, respectively. 
Applying Theorem 6.3 to $-W$, we obtain

$$
\sum_{g^{\prime} \in \operatorname{Hom}_{g}\left(\pi, G^{\prime}\right)}\left(g^{\prime}\right)^{*}([\theta])([-W])=|\Gamma| \sum_{\rho, G_{\rho} \supset g(\pi)}(|\Gamma| / \operatorname{dim} \rho)^{-\chi(W)} g^{*}\left(\zeta_{\rho}\right)([W]) .
$$

For $\theta=1$, the left-hand side is equal to $\left|\operatorname{Hom}_{g}\left(\pi, G^{\prime}\right)\right|$, and we obtain Theorem 1.1. For arbitrary $\theta$ and $g=1$, Theorem 6.3 is contained in [Tu]. A proof of Theorem 6.3 in the general case will be given elsewhere.

Theorem 6.3 yields a generalization of other formulas obtained above. We state generalizations of Formulas (4.3) and (4.6) using notation of Section 4. For a principal $G^{\prime}$-bundle $\xi^{\prime}$ on $W$, set

$$
\theta_{\xi^{\prime}}=\left(g^{\prime}\right)^{*}([\theta]) \in H^{2}\left(\pi ; \mathbb{C}^{*}\right)
$$

where $[\theta] \in H^{2}\left(G^{\prime} ; \mathbb{C}^{*}\right)$ is the cohomology class of $\theta$ and $g^{\prime}: \pi \rightarrow G^{\prime}$ is any homomorphism such that $\xi^{\prime}=\xi_{g^{\prime}}$. The cohomology class $\theta_{\xi^{\prime}}$ does not depend on the choice of $g^{\prime}$ because the conjugations in $G^{\prime}$ act trivially on $H^{*}\left(G^{\prime} ; \mathbb{C}^{*}\right)$. Theorem 6.3 implies that for the principal $G$-bundle $\xi$ over $W$ determined by a homomorphism $g: \pi \rightarrow G$,

$$
\sum_{\xi^{\prime} \in \mathcal{P}(\xi)} \frac{\theta_{\xi^{\prime}}([W])}{\left|\operatorname{Aut}_{\xi}\left(\xi^{\prime}\right)\right|}=\sum_{\rho, G_{\rho} \supset g(\pi)}\left(\frac{|\Gamma|}{\operatorname{dim} \rho}\right)^{-\chi(W)}\left(g^{*}\left(\zeta_{\rho}\right)([W])\right)^{-1},
$$

where $\rho$ ranges over the equivalence classes of irreducible $\theta$-representations of $\Gamma$ such that $G_{\rho} \supset g(\pi)$. For $G=1$, this formula boils down to

$$
\sum_{\xi^{\prime} \in \mathcal{P}} \frac{\theta_{\xi^{\prime}}([W])}{\left|\operatorname{Aut}\left(\xi^{\prime}\right)\right|}=\sum_{\rho}\left(\frac{|\Gamma|}{\operatorname{dim} \rho}\right)^{-\chi(W)},
$$

where $\rho$ ranges over the equivalence classes of irreducible $\theta$-representations of $\Gamma$.

To generalize (4.6), consider a locally trivial fiber bundle $p: E \rightarrow W$ and a cohomology class $\Theta \in H^{2}\left(E ; \mathbb{C}^{*}\right)$ whose evaluation on $\pi_{2}(E)$ is equal to 1 . Such $\Theta$ is necessarily induced from a unique element of $H^{2}\left(\pi_{1}(E) ; \mathbb{C}^{*}\right)$. We represent the latter by a $\mathbb{C}^{*}$-valued 2-cocycle $\theta$ on $\pi_{1}(E)$. Set $\pi=\pi_{1}(W)$.

Theorem 6.4. If the fiber of $p$ is path-connected and has a finite fundamental group $\Phi$, then

$$
\sum_{s \in \mathcal{S}(p)} \frac{s^{*}(\Theta)([W])}{|\operatorname{Aut}(s)|}=\sum_{\rho, \pi_{\rho}=\pi}(|\Phi| / \operatorname{dim} \rho)^{-\chi(W)}\left(\zeta_{\rho}([W])\right)^{-1},
$$

where $\rho$ runs over the equivalence classes of irreducible $\theta$-representations of $\Phi$ such that $\pi_{\rho}=\pi$.

This follows from Theorem 6.3 using the arguments of Section 4. The assumption $\Theta\left(\pi_{2}(E)\right)=1$ ensures that $s^{*}(\Theta) \in H^{2}\left(W ; \mathbb{C}^{*}\right)$ is a bubble equivalence invariant of a section $s$ so that its evaluation on $[W]$ is well-defined.

\section{References}

[FQ] G. Freed and F. Quinn, Chern-Simons theory with finite gauge group, Comm. Math. Phys. 156 (1993), 435-472.

[Fr] G. Frobenius, Über gruppencharaktere, Sitzber. Königlich Preuss. Akad. Wiss. Berlin (1896), 985-1021.

[Jo] G. Jones, Enumeration of homomorphisms and surface-coverings, Quart. J. Math. Oxford Ser. (2) 46 (1995), no. 184, 485-507. 
[Me] A. Mednykh, Determination of the number of nonequivalent coverings over a compact Riemann surface Dokl. Akad. Nauk SSSR 239 (1978), 269-271 (Russian). Soviet Math. Dokl., 19 (1978), 318-320 (English transl.).

[Se] J-P. Serre, Galois cohomology, Springer Monographs in Mathematics. Springer-Verlag, 210 p., 2002.

[Sn] N. Snyder, Mednykh's Formula via Lattice Topological Quantum Field Theories, math/0703073.

[Tu] V. Turaev, Dijkgraaf-Witten invariants of surfaces and projective representations of groups, J. of Geometry and Physics 57 (2007), 2419-2430.

Department of Mathematics, Indiana University, Bloomington IN47405, USA 